\title{
Anti-N-Methyl-D-Aspartate Receptor Encephalitis Causing a Prolonged Depressive Disorder Evolving to Inflammatory Brain Disease
}

\author{
Sara Mariotto ${ }^{a}$ Stefano Tamburin ${ }^{a} \quad$ Alessandro Salviati $^{a} \quad$ Sergio Ferrari ${ }^{a}$ \\ Marco Zoccarato $^{c}$ Bruno Giometto ${ }^{c}$ Laura Bertolasi ${ }^{a}$ \\ Franco Alessandrini ${ }^{b} \quad$ Maria Donata Benedetti ${ }^{a}$ Salvatore Monaco ${ }^{a}$ \\ ${ }^{a}$ Department of Neurological and Movement Sciences, University of Verona, and ${ }^{b}$ Division \\ of Neuroradiology, AOUI, Verona, and 'Department of Neurology, Regional Hospital 'Ca' \\ Foncello', Treviso, Italy
}

\section{Key Words}

Anti-N-methyl-D-aspartate receptor encephalitis · Optic neuritis · Depressive disorder

\begin{abstract}
Background: Anti-N-methyl-D-aspartate receptor (anti-NMDAR) encephalitis is a rapidly evolving condition that combines psychiatric and neurologic manifestations. Much remains unclear about its clinical onset and subsequent course. Although successful treatment depends on diagnosing the disorder early and therefore minimizing long-term complications, this is a difficult task owing to the atypical onset of this condition and the prolonged clinical course that has been observed in some patients. This report, illustrating a patient with slowly progressing psychiatric manifestations, unusual imaging and electrophysiological features, extends the information on varied clinical phenotypes. Case Report: A 32-year-old woman suffered from an isolated depressive disorder for 4 months before undergoing psychiatric evaluation. During the following 5 months, she manifested hypersexuality, dysarthria, imbalance, dyskinesias and decreased word output. Brain magnetic resonance imaging (MRI) showed multifocal hyperintense T2/FLAIR lesions, a few contrast-enhanced, involving the corona radiata, the periventricular white matter, the deep gray nuclei, the optic nerves and the brainstem. MRI spectroscopy disclosed confluent bilateral demyelination and focal optic nerve involvement suggesting widespread encephalitis. Visual evoked potential
\end{abstract}

Salvatore Monaco, MD

Department of Neurological and Movement Sciences

University of Verona, Policlinico G.B. Rossi, P. Ie L.A. Scuro 10

IT-37134 Verona (Italy)

E-Mail salvatore.monaco@univr.it 
studies indicated a demyelinating disorder. Serological screening and total body positronemission tomography yielded negative findings for malignancies. Cerebrospinal fluid examination disclosed IgG oligoclonal bands and anti-NMDAR antibodies. Corticosteroids and intravenous immunoglobulin provided only slight improvement, whereas switching to cyclophosphamide markedly improved her neurological status. Conclusion: In patients with a prolonged clinical course, including psychiatric and neurological symptoms, the differential diagnosis should be anti-NMDAR encephalitis. This report expands the known disease phenotypes in this emerging condition.

(C) 2014 S. Karger AG, Basel

\section{Introduction}

Anti-N-methyl-D-aspartate receptor (anti-NMDAR) encephalitis is a newly recognized antibody-mediated disorder, arising in a paraneoplastic or non-paraneoplastic setting. In about $70 \%$ of patients, the disorder begins with a prodrome, characterized by fever, headache with stiff neck, malaise and gastrointestinal symptoms. Later on, patients typically manifest combined mood and behavioral changes such as thought disorders, psychosis, staring and unresponsiveness, rapidly evolving to generalized seizures, facial dyskinesias, pathologic posturing, speech dysfunction, autonomic instability and hypoventilation. Electroencephalographic (EEG) tracings usually show a nonspecific, generalized slowing or epileptiform activity. Despite this deleterious clinical state, brain magnetic resonance imaging (MRI) is unremarkable in $50-77 \%$ of patients or may show mild and transient nonspecific abnormalities in various brain regions [1]. Brain positron-emission tomography (PET) may disclose hypo- or hypermetabolism in cortical areas and the subcortical nuclei, a finding that, if combined with negative MRI imaging, may help as a guide in the diagnosis [2]. Confirmation tests for diagnosing anti-NMDAR encephalitis require indirect immunofluorescence and cell-based assay. We herein describe a patient with anti-NMDAR encephalitis, with an atypical, slowly evolving clinical course and unusual neurophysiological and radiological findings.

\section{Case Report}

In June 2012, a 32-year-old woman was referred to our clinic to investigate a progressive cognitive dysfunction, cerebellar ataxia, abnormal movements and a reduced word output. She had a 9-month history of slowly progressing mood changes and 'brain fog'. The patient was a high school teacher, with an unremarkable medical history, who lived with her husband and 20-month-old son. Five months earlier, she had attended a psychiatric clinic where she received a tentative diagnosis of postpartum depression. Antidepressants and benzodiazepines left her clinical condition unchanged. Thereafter, while under psychiatric care, the patient slowly began to experience hypersexuality, dysarthria, imbalance, decreased word output and episodes of unexplained crying. EEG tracings at that time showed diffuse slowing without epileptiform activity. Upon referral to our clinic, a brain MRI disclosed widespread multifocal lesions, hyperintense on T2-weighted images, involving the brainstem, the thalamus, the caudate and lenticular nuclei, the insula and the corona radiata. Two periventricular lesions and several small spots in the insula and pyramidal tract showed a contrast enhancement. Given the clinical and MRI findings, the patient was admitted for further investigation. 
On examination, the patient was alert. Her speech was dysarthric, hypophonic and she answered in short phrases. Cognitive testing disclosed a markedly impaired executive function. Color vision testing detected dyschromatopsia and visual field testing found full visual fields on confrontation. Saccades were hypometric and slow with upward palsy. She had postural instability, an ataxic gait, dysmetria, oro-lingual facial dyskinesias, choreoathetosis, hand dystonia and mild muscle rigidity. Deep tendon reflexes were brisk with bilateral ankle clonus. Hematologic and serum chemical tests, including a screening for rheumatologic diseases and a comprehensive panel for infectious, metabolic and paraneoplastic disorders yielded negative findings. The whole-body PET scan was unremarkable. Upper and lower limb somatosensory and motor evoked potentials elicited slightly reduced amplitudes on the left side; conversely, flash-evoked visual potentials showed prolonged N2 and P2 latencies (fig. 1). Cerebrospinal fluid (CSF) analysis, including tau protein level and a 14-3-3 protein assay yielded normal findings, except for an elevated IgG index and oligoclonal IgG bands; we detected anti-NMDAR IgG antibodies. CSF cell count showed 1 white cell per milliliter and nucleic acid testing for HSV, VZV and enteroviruses was negative. In addition, testing of serum and CSF for water channel protein aquaporin-4-IgG-Ab by indirect immunofluorescence was negative. The positive CSF anti-NMDAR and negative serum findings were also confirmed elsewhere using human embryonic kidney cells expressing the NR1 subunits (Dr. Josep Dalmau, Hospital Clinic, Universitat de Barcelona, Spain). In July 2012, a brain scan using a 3T MR scanner detected multiple and partially confluent punctate foci of T2/FLAIR hyperintensity, unenhanced after gadolinium, bilaterally in the basal telencephalon, diencephalon, external capsulae, subcortical insula, periventricular white matter, temporal horns, corpus callosum, and optic nerves as well as the ventral cerebral peduncles, pontinemesencephalic junction and dorsal pons (fig. 2). Spectroscopy of the left basal ganglia region and the right midbrain tegmentum, acquired with the single-slice multi-voxel chemical-shift technique (TE $=30$ and $135 \mathrm{~ms}$ ), showed a reduced $N$-acetyl aspartate (NAA) peak and a slightly increased choline (Cho) peak, resulting in an inverted Cho/NAA ratio, in addition to lipid traces and an absence of lactate within a high signal-intensity foci.

These brain metabolic findings were consistent with widespread encephalitis, characterized by confluent bilateral demyelination areas and focal optic nerve involvement in a late subacute stage. The patient was treated with oral corticosteroids followed by two IVIg cycles, given at 2-week intervals, in August 2012. After treatment, the gait ataxia improved and her mood improved, but the EEG tracings were unchanged. At neuropsychological evaluation, working memory, attention, and the logical-deductive capacities remained altered; the Mini-Mental State Examination score was 26/30. Between August and November 2012, the patient received 4 IVIg cycles given at 4-week intervals.

In November, a repeat MRI scan showed a reduction in the number and the extension of lesions previously described, with a new, non-enhancing lesion in the left basal ganglia. Thereafter, therapy was switched to cyclophosphamide. In December 2012, neurological evaluation showed a slight improvement of speech, ataxia and cognitive function; dystonic posturing of the left hand, slight postural tremor of the left limbs, brisk reflexes, muscle rigidity in the lower limbs and oro-lingual dyskinesias were observed. In March 2013, brain MRI showed that most alterations had disappeared and residual lesions were no longer contrast enhanced (fig. 2). A PET brain scan showed a reduced glucose metabolism in the temporal lobes and the left striatum, relative to other cortical areas (fig. 3).

At the last visit in August 2013, cognitive function, speech and balance had improved, but emotional liability and her attention deficit persisted. 
Mariotto et al.: Anti-N-Methyl-D-Aspartate Receptor Encephalitis Causing a Prolonged Depressive Disorder Evolving to Inflammatory Brain Disease

\section{Discussion}

The case we reported shows that anti-NMDAR encephalitis may present with longstanding emotional symptoms, therefore suggesting a primary psychiatric disorder, as opposed to the more commonly observed acute onset progression. This presentation is challenging for clinicians because neuropsychiatric symptoms, including behavioral disturbances, delusions and hallucinations, progress rapidly in typical cases toward staring unresponsiveness within days to weeks. Our patient's neuropsychiatric manifestations progressed to neurological dysfunction relatively late ( 9 months after presentation). The slow progression over several months suggested a different diagnosis other than a depressive disorder, among them anti-NMDAR encephalitis.

Additional atypical findings in the present case included demyelinating optic neuritis, a condition anecdotally reported in anti-NMDAR encephalitis [3, 4], and inflammatory demyelinating lesions. In the setting of neurological paraneoplastic/dysimmune disorders, cellular inflammation usually arises in conditions associated with antibodies against intracellular antigens, whereas cell-mediated immunity plays a minor role in cases triggered by antibodies against neuronal surface epitopes [5]. Accordingly, typical pathological findings in anti-NMDAR encephalitis consist of IgG deposition and microgliosis without complement activation and cellular inflammation. Necrotic cystic lesions have been reported in atypical forms with striatal or cortical involvement [6]; only in a few cases, minimal T- and B- cellular infiltration has been detected in the hippocampus, the forebrain, the basal ganglia and the spinal cord [7,8]. Conversely, consistent mixed perivascular and parenchymal cellular infiltrates have been pathologically observed in a patient with anti-NMDAR antibodies and seronegative optic neuromyelitis [3]. However, the number of cases with MRI evidence of inflammatory demyelinating lesions, similar to that reported here, is more ample, suggesting that the pathogenesis of anti-NMDAR demyelinating variants and optic neuritis involves cell-mediated mechanisms $[3,4,9]$. Finally, although we obtained the FDGPET study at a stage when MRI detected no active inflammatory lesions, we suggest that the left striatal hypometabolism could reflect a post-inflammatory defect rather than a functional hypoglutamatergic state. This conclusion goes against the increased glucose uptake observed in several investigated cases. As our patient's marked improvement after cyclophosphamide treatment shows, assessing how cellular inflammation contributes in anti-NMDAR encephalitis is important in choosing the therapeutic approach because a plasma exchange and IVIg are effective in typical cases, but not in demyelinating forms and inflammatory variants.

\section{Disclosure Statement}

This work was supported in part by the grant 'Disabilità cognitiva e comportamentale nelle demenze e nelle psicosi' from the Fondazione Cariverona to Salvatore Monaco. The authors declare no conflict of interest. 


\section{Case Reports in Neurology}

\begin{tabular}{l|l}
\hline Case Rep Neurol 2014;6:38-43 & \\
\hline DOI: 10.1159/000358820 & $\begin{array}{l}\text { ○ 2014 S. Karger AG, Basel } \\
\text { www.karger.com/crn }\end{array}$ \\
\hline
\end{tabular}

Mariotto et al:: Anti-N-Methyl-D-Aspartate Receptor Encephalitis Causing a Prolonged Depressive Disorder Evolving to Inflammatory Brain Disease

\section{References}

1 Dalmau J, Lancaster E, Martinez-Hernandez E, Rosenfeld MR, Balice-Gordon R: Clinical experience and laboratory investigations in patients with anti-NMDAR encephalitis. Lancet Neurol 2011;10:63-74.

2 Mohr BC, Minoshima S: F-18 fluorodeoxyglucose PET/CT findings in a case of anti-NMDA receptor encephalitis. Clin Nucl Med 2010;35:461-463.

-3 Kruer MC, Koch TK, Bourdette DN, Chabas D, Waubant E, Mueller S, Moscarello MA, Dalmau J, Woltjer RL, Adamus G: NMDA receptor encephalitis mimicking seronegative neuromyelitis optica. Neurology 2010;74:1473-1475.

4 Ishikawa N, Tajima G, Hyodo S, Takahashi Y, Kobayashi M: Detection of autoantibodies against NMDA-type glutamate receptor in a patient with recurrent optic neuritis and transient cerebral lesions. Neuropediatrics 2007;38:257-260.

5 Graus F, Saiz A, Dalmau J: Antibodies and neuronal autoimmune disorders of the CNS. J Neurol 2010;257:509-517.

6 Tzoulis C, Vedeler C, Haugen M, Storstein A, Tran GT, Gjerde IO, Biermann M, Schwarzlmuller T, Bindoff LA: Progressive striatal necrosis associated with anti-NMDA receptor antibodies. BMC Neurol 2013;13:55.

7 Camdessanché JP, Streichenberger N, Cavillon G, Rogemond V, Jousserand G, Honnorat J, Convers P, Antoine JC: Brain immunohistopathological study in a patient with anti-NMDAR encephalitis. Eur J Neurol 2011;18:929-931.

-8 Tüzün E, Zhou L, Baehring JM, Bannykh S, Rosenfeld MR, Dalmau J: Evidence for antibody-mediated pathogenesis in anti-NMDAR encephalitis associated with ovarian teratoma. Acta Neuropathol 2009;118:737-743.

-9 Lekoubou A, Viaccoz A, Didelot A, Anastasi A, Marignier R, Ducray F, Rogemond V, Honnorat: Anti-N-methylD-aspartate receptor encephalitis with acute disseminated encephalomyelitis-like MRI features. Eur J Neurol 2012;19:e16-e17.
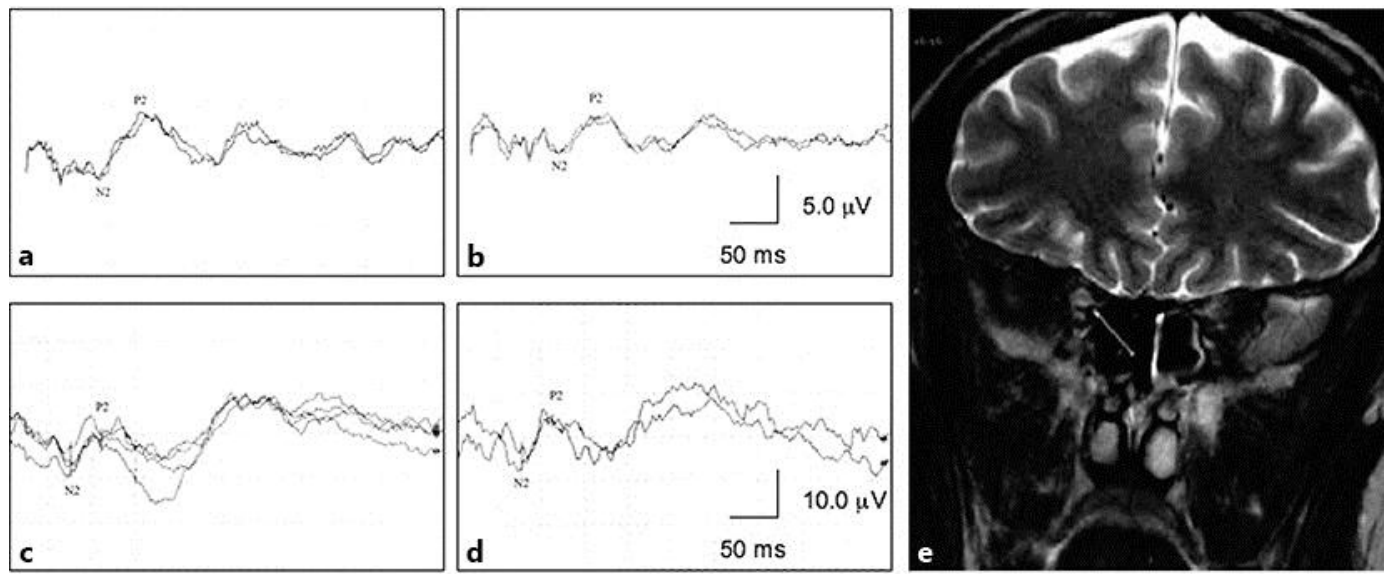

Fig. 1. Flash-evoked visual potentials show prolonged N2 and P2 latencies in both sides at 9 months after disease onset $(\mathbf{a}, \mathbf{b})$; marked improvement 9 months later $(\mathbf{c}, \mathbf{d})$. 3T MRI coronal T2-weighted images show increased signals in both optic nerves, more prominent on the right (e). 


\section{Case Reports in Neurology}
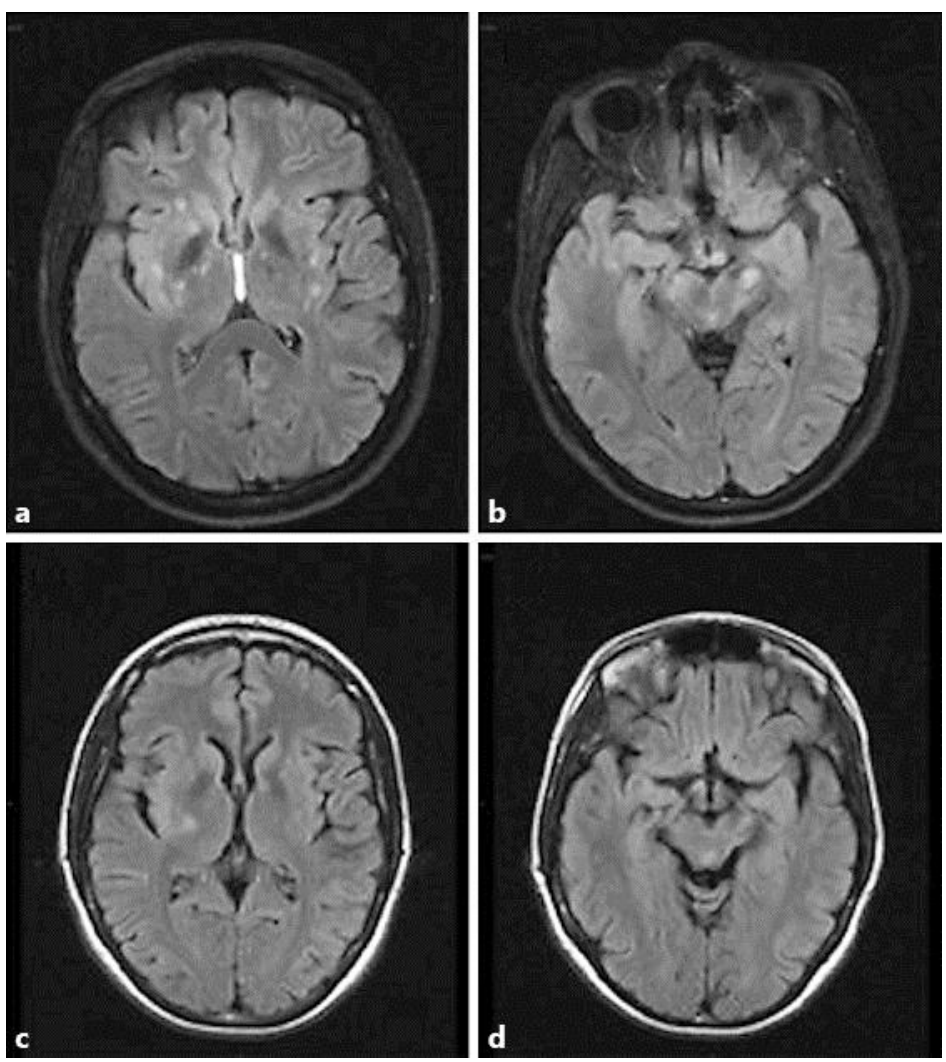

\begin{tabular}{l|l}
\hline DOI: 10.1159/000358820 & $\begin{array}{l}\text { ○ 2014 S. Karger AG, Basel } \\
\text { www.karger.com/crn }\end{array}$ \\
\hline
\end{tabular}

Mariotto et al:: Anti-N-Methyl-D-Aspartate Receptor Encephalitis Causing a Prolonged Depressive Disorder Evolving to Inflammatory Brain Disease

Fig. 2. Brain MRI at neurological presentation shows multifocal hyperintense lesions within the basal ganglia (a) and midbrain (b); significantly reduced lesion load after 9 months (c, d).
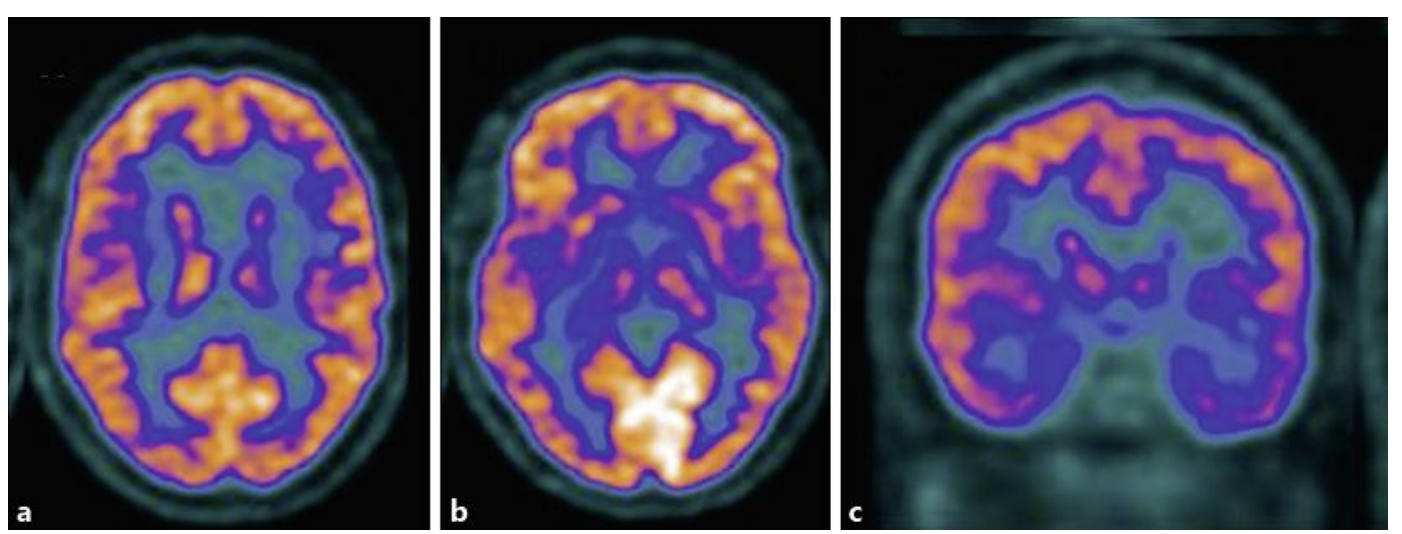

Fig. 3. Axial (a, b) and coronal (c) brain 18F-FDG PET slices showing reduced glucose uptake in the left caudate nucleus, putamen, thalamus and bilateral mesial temporal lobes. 